Pacific Journal of Mathematic 


\title{
SUBGROUPS OF FINITE INDEX IN PROFINITE GROUPS
}

\author{
Michael P. Anderson
}

A profinite group is called strongly complete if every subgroup of finite index is open and of type (AF) if it has only finitely many subgroups of any fixed index. In this paper it is shown that a topologically finitely generated abelian by pro-nilpotent profinite group is strongly complete, and that a pro-solvable profinite group is strongly complete if is of type (AF).

In [6] it is shown that every strongly complete group is of type (AF). The proof of the converse of this result for pro-solvable groups rests on two facts. The first is that any finite quotient of a pro-solvable profinite group is solvable. The second is that any measurable subgroup of finite index in a profinite group is open. In particular, any verbal subgroup of finite index is open.

Lazard ([4], Chap. III, 3.) has shown that every pro-p-group which admits a $p$-adic analytic structure is strongly complete and Serre [8] has extended this result to all topologically finitely generated pro- $p$-groups. The proof given here that topologically finitely generated abelian by pro-nilpotent groups are strongly complete combines Serre's technique with a similar technique used to prove the result for metabelian profinite groups. All these proofs rest on the fact that the commutator subgroup of the group in question is closed.

The paper is concluded with some examples and a remark on the relationship of the problems considered here with some problems in the theory of finite groups.

I would like to thank Prof. Walter Feit and Prof. Roger Howe for discussions on this work. I would also like to thank the referee for suggesting that Theorem 3 be strengthened to include Serre's result and for forwarding the letter of Serre to me.

1. Abstract properties of profinite groups. Let $G$ be a profinite group. We will denote the order of $G$ (cf. [7]) by $o(G)$. If $x$ is an element of $G$ we define the exponent of $x$ to be the order of the closed subgroup of $G$ generated by $x$.

Let $\hat{G}$ be the completion of the abstract group $G$ for the topology having as neighborhood basis of the identity the subgroups of finite index. There is a natural homomorphism $G \rightarrow \hat{G}$. Since $\hat{G}$ is universal for continuous homomorphisms of the discrete group $G$ to profinite 
groups, we have a natural continuous homomorphism $\hat{G} \rightarrow G$ where $G$ is given its initial profinite topology. The reader may easily verify the following result.

PROPOSITION 1. There is an exact sequence of profinite groups and continuous homomorphisms $\langle e\rangle \rightarrow K \rightarrow \hat{G} \rightarrow G \rightarrow\langle e\rangle$. Moreover the canonical homomorphism $G \rightarrow \hat{G}$ splits this sequence and is continuous if and only if $K=e$ i.e. $G$ is strongly complete.

In view of the above proposition it makes sense to ask if an abstract group is strongly complete.

We now verify that an extension of two strongly complete groups is strongly complete. The same result holds for groups of type (AF).

Proposition 2. Let $\langle e\rangle \rightarrow G^{\prime} \rightarrow G \rightarrow G^{\prime \prime} \rightarrow\langle e\rangle$ be an exact sequence of groups. If $G^{\prime}$ and $G^{\prime \prime}$ are strongly complete, then $G$ is strongly complete. If $G$ is a strongly complete profinite group and $G^{\prime}$ is a closed subgroup, then $G^{\prime \prime}$ is strongly complete.

Proof. The homomorphisms $G^{\prime} \rightarrow \hat{G}^{\prime}$ and $G^{\prime \prime} \rightarrow \hat{G}^{\prime \prime}$ are isomorphisms by assumption. Thus by [1] Proposition 2 the injective map $G \rightarrow \hat{G}$ must be surjective and so an isomorphism. A similar argument proves the second assertion.

Let $G$ and $G^{\prime}$ be profinite groups such that $G$ is strongly complete, and let $h: G \rightarrow G^{\prime}$ be a homomorphism. The inverse image of any open subgroup of $G^{\prime}$ under $h$ is of finite index in $G$. Since $G$ is strongly complete, this subgroup must be open and thus $h$ must be continuous. We have proven.

Proposition 3. Let $G$ and $G^{\prime}$ be profinite groups such that $G$ is strongly complete. Then any homomorphism $G \rightarrow G^{\prime}$ is continuous.

COROLlARY. Let $G$ be strongly complete and $M$ a countable residually finite group (e.g. the group of integral points of a matrix group over a number field). Then any homomorphism $G \rightarrow M$ has finite image.

Proof. Since $M$ is residually finite, the image of $G \rightarrow M$ is the same as the image of $G \rightarrow \hat{M}$. By Proposition $3, G \rightarrow \hat{M}$ is continuous and thus has closed image. Since $M$ is countable, the image is at most a countable set. Thus the image is a finite set.

The next theorem shows that the order of a profinite group is an invariant of the underlying abstract group.

THEOREM 1. Let $G$ be a profinite group and $G^{\prime} \subset G$ a subgroup of finite index. Then $\left[G: G^{\prime}\right]$ divides $o(G)$ and thus $o(G)=o(G)$. 
Proof. It suffices to check the first statement for $G^{\prime}$ a normal subgroup of finite index. Let $p$ be a prime dividing the order of the finite group $G / G^{\prime}$, and let $x$ be an element of $G / G^{\prime}$ such that $x$ is of exponent $p$. If $y$ is an element of $G$ projecting to $x$, then, since topologically finitely generated profinite abelian groups are strongly complete, $p$ divides the exponent of $y$ and thus divides $o(G)$. If $o(G)$ is divisible by an infinite power of $p$, we are done. If the maximal power of $p$ dividing $o(G)$ is $p^{n}$ with $n$ finite, we can find an open subgroup $U \subset G$ such that $(p, o(U))=1$. But then by the above argument, $\left(p,\left[U: U \cap G^{\prime}\right]\right)=1$ and thus $p^{n}$ divides $[G: U]$.

REMARK. One should perhaps compare the above result with the fact that a compact group is connected if and only if it is divisible. In this regard note also that the above argument shows that an element $x$ in $G$ is contained in a Sylow $p$-subgroup of $G$ if and only if it is $n$-divisible for all $(n, p)=1$.

COROLlaRY. Let $G$ be a profinite group such that for all primes $p$ the Sylow p-subgroups of $G$ are finite. Then $G$ is strongly complete.

The next result will give the criterion used in the following section to determine when subgroups of finite index in pro-solvable profinite groups are open. We let $\mu$ denote the normalized Haar measure on the compact group $G$. We refer to [5] for the definition of a verbal subgroup.

Proposition 4. Let $G$ be a profinite group and $G^{\prime}$ a verbal subgroup of $G$. Then $G^{\prime}$ is open in $G$ if and only if it is of finite index.

Proof. Any open subgroup in $G$ is of finite index, so it suffices to check that if $G^{\prime}$ is of finite index, then it is open. $G^{\prime}$ is a countable union of closed subsets of $G$. Thus $G^{\prime}$ is a Borel set and in particular is measurable. If $G^{\prime}$ is of finite index, $\mu\left(G^{\prime}\right)>0$ because $G$ is covered by a finite number of translates of $G^{\prime}$. But then $G^{\prime}$ must contain an open subgroup of $G$ (cf. [2] pg. 296) and thus must itself be open.

If $G$ is a profinite group, we let $\operatorname{Hom}(G, Z / n Z)$ denote the set of homomorphisms from $G$ to the cyclic group of order $n$ and $\mathrm{Hom}_{\text {cont }}(G, Z / n Z)$ denote the subset of continuous homomorphisms.

Proposition 5. Let $G$ be a profinite group such that the set $\operatorname{Hom}(G, Z / n Z)$ is finite. Then $\operatorname{Hom}_{\text {cont }}(G, Z / n Z)=\operatorname{Hom}(G, Z / n Z)$ i.e. every homomophism is continuous.

Proof. Let $G^{\prime}$ be the verbal subgroup of $G$ generated by the words 
$[X, Y]=X^{-1} Y^{-1} X Y$ and $X^{n}$. Then $G^{\prime}$ is the intersection of the kernels of all the homomorphisms from $G$ to $Z / n Z$. By assumption $G^{\prime}$ is of finite index in $G$, and thus by Proposition $4, G^{\prime}$ is open in $G$. Thus the result follows.

2. Pro-solvable profinite groups. We say that a profinite group is pro-solvable if it is the inverse limit of finite solvable groups. If $G$ is a pro-finite group and $p$ is a prime number, we define a Sylow $p$-complement in $G$ to be a closed subgroup $P^{\prime} \subset G$ such that $p$ does not divide $o\left(P^{\prime}\right)$ and $\left[G: P^{\prime}\right]$ is a power of $p$. The following is a straightforward profinite extension of a theorem of P. Hall (cf. [3] pp. 185-189).

Proposition 6. A profinite group $G$ is pro-solvable if and only if $G$ has a Sylow p-complement for each prime $p$. If $P$ is a Sylow p-subgroup and $P^{\prime}$ is a Sylow p-complement, then $G=P P^{\prime}$ i.e. every element $x$ in $G$ admits a unique representation $x=y z$ with $y$ in $P$ and $z$ in $P^{\prime}$.

A direct consequence of this is the following proposition.

Proposition 7. Let $G$ be a pro-solvable group and $G^{\prime} \subset G$ a norn al subgroup of finite index. Then $G / G^{\prime}$ is a finite solvable group.

Proof. We will verify that $G / G^{\prime}$ has a Sylow $p$-complement for each $p$. Let $P$ be a Sylow $p$-subgroup of $G$ and $P^{\prime}$ a Sylow $p$ complement. Then

$$
\left[G: G^{\prime}\right]=\left[P: P \cap G^{\prime}\right]\left[P^{\prime}: P^{\prime} \cap G^{\prime}\right] .
$$

By Theorem 1, $\left[P: P \cap G^{\prime}\right]$ is a power of $p$ and $\left[P^{\prime}: P^{\prime} \cap G^{\prime}\right]$ is prime to p. Thus $P^{\prime} / P^{\prime} \cap G^{\prime}$ is a Sylow $p$-complement in $G / G^{\prime}$.

Corollary. A profinite group $G$ is pro-solvable if and only if $\hat{G}$ is pro-solvable.

We are now ready to prove the following.

THEOREM 2. A pro-solvable profinite group is strongly complete if and only if it is of type (AF).

Proof. As was pointed out in the introduction, the fact that a strongly complete group is of type (AF) was shown by Peterson in [6]. In the case of a pro-solvable group the essential fact is that the double dual of an infinite dimensional vector space over a finite field is strictly larger than the dimension of the space. To prove the converse 
implication we use Proposition 5 and Proposition 7 and argue by induction. Let $G^{\prime}$ be a normal subgroup of finite index in $G$. $G / G^{\prime}$ is solvable by Proposition 7. If $G / G^{\prime}$ is abelian, then $G^{\prime}$ is open by Proposition 5. Now use induction on the length of the derived series of $G / G^{\prime}$.

We will now prove a theorem giving a sufficient condition for a group to be strongly complete. After some preliminary conventions, the proof begins with a lemma on commutators.

Let $G$ be a profinite group and $N$ a closed normal subgroup of $G$. For $g$ and $g^{\prime}$ elements of $G$ we use the notation

$$
g \equiv g^{\prime} \operatorname{Mod} N
$$

to mean that $g=g^{\prime} n$ where $n$ is an element of $N$. We denote the closure of the commutator group $[G, N]$ in $N$ by $\overline{[G, N]}=\overline{[G, N]_{1}}$ and we define inductively $\overline{[G, N]}_{k+1}=\left[G, \overline{[G, N]}_{k}\right]$. We define $\overline{[G, N]_{\infty}}=$

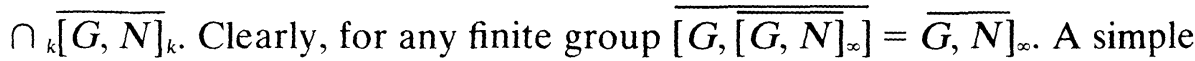
argument, which will be left to the reader, shows that the same result is true for any profinite group $G$.

A profinite group is called pro-nilpotent if it is the inverse limit of finite nilpotent groups. $G$ is pro-nilpotent if and only if $\overline{[G, G]_{\infty}}=$ $\langle e\rangle$. We say that a profinite group $G$ is abelian by pro-nilpotent if $G$ has a closed normal abelian subgroup $A$, such that $G / A$ is pro-nilpotent. $G$ is abelian by pro-nilpotent if and only if $\overline{[G, G]_{\infty}}$ is abelain.

LEMma. Let $G$ be a profinite group with topological generators $x_{1}, \cdots, x_{d}$, and let $N$ be a normal subgroup of $G$. Then for any $w$ in $\overline{[G, N]}$ and every $k \geqq \infty$ there exist $n_{1}, \cdots, n_{d}$ in $N$ such that:

$(1)_{k} \quad w \equiv\left[x_{1}, n_{1}\right] \cdots\left[x_{d}, n_{d}\right] \operatorname{Mod} \overline{\left[N, \overline{[G, N]}_{k}\right]}$

$(2)_{k} \quad w \equiv\left[x_{1}, n_{1}\right] \cdots\left[x_{d}, n_{d}\right] \operatorname{Mod} \overline{\left.[\overline{[G, N]}, \overline{[G, N]}]_{k}\right]}$ and there exist $m_{1}, \cdots, m_{d}$ in $\overline{[G, N]}_{\infty}$ such that

(3) $w \equiv\left[x_{1}, n_{1}\right] \cdots\left[x_{d}, n_{d}\right]$

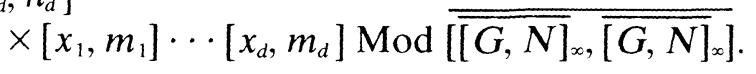

Proof. We will proceed by induction starting with (1), For all $x$ and $y$ in $G$ and $n$ and $m$ in $N$ we have the following commutator formulas:

(0) $[x, y]=x^{-1} y^{-1} x y$

(i) $\quad[x y, n]=\left[y, n^{x}\right][x, n]$

(ii) $\left[x^{-1}, n\right]=\left[n^{x^{-1}}, x\right]$ 
(iii) $\left.[n, x]=\left[x, n^{-1}\right]\left[[x, n], n^{-1}\right] \equiv\left[x, n^{-1}\right] \operatorname{Mod} \overline{[N, \overline{[G, N]}}\right]$

(iv) $[n, n]\left[y, n^{\prime}\right] \equiv\left[y, n^{\prime}\right][x, n] \operatorname{Mod} \overline{[\overline{[G, N]}, \overline{[G, N]}]}$

(v) $\left[x, n^{\prime}\right][x, n] \equiv\left[x, n n^{\prime}\right]\left[n^{\prime},[x, n]\right] \equiv\left[x, n n^{\prime}\right] \operatorname{Mod} \overline{[N, \overline{[G, N]}}$.

The $x_{\imath}$ generate a dense subgroup $G^{\prime} \subset G$, and the commutator

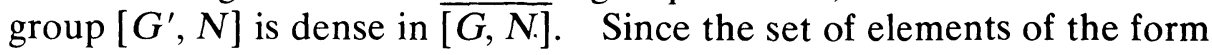
$\left[x_{1}, n_{1}\right] \cdots\left[x_{d}, n_{d}\right] w^{\prime}$ with $n$ in $N$ and $w^{\prime}$ in $\overline{[N, \overline{[G, N]}]}$ is closed, it suffices to prove the result for $w$ in $\left[G^{\prime}, N\right]$ i.e. for finite products of elements of the form $\left[g^{\prime}, n\right]$ and $\left[n, g^{\prime}\right]$, where $g^{\prime}$ is a finite product of elements of the form $x_{\imath}$ and $x_{\imath}^{-1}$. But by (i)-(iii) any basic commutator can be put in the required form and by (iii)-(v) the elements of the required form constitute a subgroup. Thus $(1)_{1}$ is proven.

Now we will show that $(1)_{k}$ implies $(1)_{k+1}$ and $(2)_{k}$. By hypothesis any $w$ in $\overline{[G, N]}$ is of the form $\left[x_{1}, n_{1}\right] \cdots\left[x_{d}, n_{d}\right] w^{\prime}$ with $w^{\prime}$ in $\overline{\left[N, \overline{[G, N]}_{k}\right]}$. But $\overline{[N, \overline{[G, N]}]}$ is contained in $\overline{\left[G, \overline{[G, N]_{k}}\right]}$ and thus taking $N$ to be ${\overline{[G, N]_{k}}}_{k}$ in (1) we see that there exist $m_{i}$ in $[G, N]_{k}$ such that

$$
w^{\prime} \equiv\left[x_{1}, m_{1}\right] \cdots\left[x_{d}, m_{d}\right] \operatorname{Mod} \overline{\left[\overline{[G, N]}_{k}, \overline{\left[G, \overline{[G, N]}_{k}\right]}\right]}
$$

and thus

$$
\left.w \equiv\left[x_{1}, n_{1}\right] \cdots\left[x_{d}, n_{d}\right]\left[x_{1}, m_{1}\right] \cdots\left[x_{d}, m_{d}\right] \operatorname{Mod} \overline{[\overline{[G, N]}},\left[G, \overline{[G, N]}_{k}\right]\right] .
$$

For $x$ and $y$ in $G, n$ in $N$, and $m$ in $\overline{[G, N]}_{k}$ we have the following commutator formulas:

(i) $\quad[x, n][y, m] \equiv[y, m][x, n] \operatorname{Mod} \overline{[\overline{[G, N]}, \overline{[G, N]}} \overline{k+1}]$

(ii) $[x, n][x, m] \equiv[x, n m] \operatorname{Mod} \overline{\left[N, \overline{[G, N]}_{k+1}\right]}$

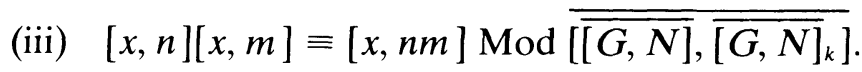

By (i) and (ii) we have

$$
w \equiv\left[x_{1}, n_{1} m_{1}\right] \cdots\left[x_{d}, n_{d} m_{d}\right] \operatorname{Mod} \overline{\left[N, \overline{[G, N]}_{k+1}\right]}
$$

and thus $(1)_{k+1}$ is proven. By (i) and (iii) we have

$$
w \equiv\left[x_{1}, n_{1} m_{1}\right] \cdots\left[x_{d}, n_{d} m_{d}\right] \operatorname{Mod} \overline{[\overline{[G, N]}, \overline{[G, N]}} \overline{[G}_{k}
$$

and thus $(2)_{k}$ is proven. 
We will now demonstrate (3), (2) $)_{\infty}$ and (1) $)_{\infty}$ For each $k$ choose $n_{t, k}$ in $N$ such that

$$
w \equiv\left[x_{1}, n_{1, k}\right] \cdots\left[x_{d}, n_{d, k}\right] \operatorname{Mod} \overline{\left[N, \overline{[G, N]}_{k}\right]}
$$

For each $i$ choose an accumulation point $n_{l}$ of the sequence $n_{l, k}$. Then for all $l \geqq k$ we have

$$
w \equiv\left[x_{1}, n_{1, l}\right] \cdots\left[x_{d}, n_{d, l}\right] \operatorname{Mod} \overline{\left[N, \overline{[G, N]}_{k}\right]}
$$

Thus by continuity of the group operations, we have for all $k$.

$$
w \equiv\left[x_{1}, n_{1}\right] \cdots\left[x_{d}, n_{d}\right] \operatorname{Mod} \overline{\left[N, \overline{[G, N]}_{k}\right]} .
$$

Therefore $w=\left[x_{1}, n_{1}\right] \cdots\left[x_{d}, n_{d}\right] w^{\prime}$ where $w^{\prime}$ lies in $\bigcap_{k} \overline{[N, \overline{[G, N]}]_{k}}$ and thus in $\overline{[G, N]}_{\infty}$. As was remarked previously, $\overline{\left.[G, \overline{[G, N]}]_{\infty}\right]}=$

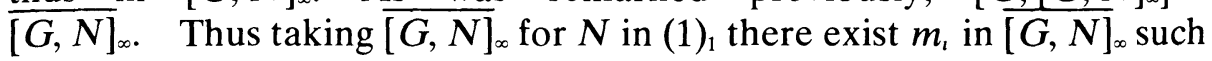
that

$$
w^{\prime} \equiv\left[x_{1}, m_{1}\right] \cdots\left[x_{d}, m_{d}\right] \operatorname{Mod} \overline{\left.\left.[\overline{[G, N]}]_{\infty}, \overline{[G, N]}\right]_{\infty}\right]}
$$

Therefore

$$
w \equiv\left[x_{1}, n_{1}\right] \cdots\left[x_{d}, n_{d}\right]\left[x_{1}, m_{1}\right] \cdots\left[x_{d}, m_{d}\right] \operatorname{Mod} \overline{\left.[\overline{[G, N]}]_{\infty}, \overline{[G, N]_{\infty}}\right]}
$$

which demonstrates (3).

For $x$ and $y$ in $G, n$ in $N$, and $m$ in $\overline{[G, N]}_{\infty}$ we have:

(i) $[x, n][y, m] \equiv[y, n][x, n] \operatorname{Mod} \overline{\left.[\overline{[G, N]}, \overline{[G, N]}]_{\infty}\right]}$

(ii) $[x, n][x, m] \equiv[x, n m] \operatorname{Mod} \overline{[\overline{[G, N]}, \overline{[G, N]}]_{\infty}}$.

Therefore

$$
\left.w \equiv\left[x_{1}, n_{1} m_{1}\right] \cdots\left[x_{d}, n_{d} m_{d}\right] \operatorname{Mod}[\overline{[G, N]}, \overline{[G, N]}]_{\infty}\right]
$$

which demonstrates $(2)_{\infty}$ and thus (1)

The lemma will be used in the proof of the next theorem through the following result.

LemMA'. Let $G$ and $N$ be as above and assume $\overline{[G, N]}_{\infty}$ is abelian. Then $[G, N]$ is closed. 
Proof. By (3), every element of $\overline{[G, N]}$ is the product of at most $2 d$ basic commutators $[g, n]$, and thus $\overline{[G, N]}$ is contained in $[G, N]$.

REMARK. In a preliminary version of this paper the above Lemma

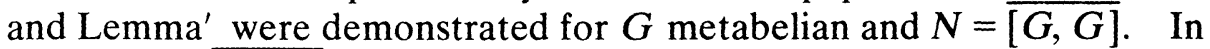
this case $[N, \overline{[G, N]}]=\langle e\rangle$ and so everything reduces to $(1)_{\infty}$. In [8] Serre proves the above Lemma and Lemma' in the case $G$ a pro- $p$-group and $N=G$ and he remarks that Lemma' is true for $N=[G, G]_{k}$. In this case $\overline{[G, G]_{\infty}}=\langle e\rangle$ so that everything reduces to (1) . The idea of Serre's proof is essentially the same as above but uses the language of Lie algebras. Combining (1) with $N=G$ and (1) with $N=[G, G]_{\infty}$ enables us to prove Theorem 3 .

We now state the theorem.

THEOREM 3. A topologically finitely generated abelian by pronilpotent group is strongly complete.

Proof. Let $G$ be a topologically finitely generated profinite group and $G^{\prime}$ a normal subgroup of finite index in $G$. By Proposition $7, G / G^{\prime}$ is solvable. Since any open subgroup of $G$ is topologically finitely generated and abelian by pro-nilpotnet, by induction on the length of the derived series of $G / G^{\prime}$ it suffices to consider $G^{\prime}$ such that $G / G^{\prime}$ is abelian. Since topologically finitely generated abelian profinite groups are strongly complete, it suffices to show that $G^{\prime}$ contains the closure of the commutator subgroup of $G$. But by hypothesis $\overline{[G, G]}_{\infty}$ is abelian, and thus by Lemma $[G, G]$ is closed.

We now derive a corollary which is a slight improvement of Serre's result. We recall that a profinite group is said to be of type $(F)$ if it has only finitely many open subgroups of any fixed index (cf. [7]). In general this condition is weaker than being topologically finitely generated but for pro- $p$-groups they are equivalent.

COROLlaRY. A pro-nilpotent profinite group is strongly complete if and only if it is of type (F).

Proof. Arguing as before, we may reduce to the case where $G^{\prime}$ is a normal subgroup of prime index in $G$. $G=G_{p} \times G_{p^{\prime}}$ where $G_{p}$ is the unique Sylow $p$ subgroup of $G$, and $G_{p^{\prime}}$ is the unique Sylow $p$ complement of $G_{p}$. Since $G_{p^{\prime}}$ has order prime to $p, G^{\prime}$ contains $G_{p^{\prime}}$, and it suffices to show $G^{\prime} \cap G_{p}$ is open in $G_{p}$. But $G_{p}$ is a pro-p-group of type (F). Thus $G_{p}$ is topologically finitely generated and the result follows from Theorem 3 .

REMARK. In the above it was seen that the strong completeness of 
finitely generated pro-solvable groups can be deduced from the closedness of their commutator subgroups, and that this reduces to finding a bound on the number of basic commutators one must multiply to get an element of the commutator subgroup. In a certain sense, the converse is also true. For instance, all topologically finitely generated pro-solvable profinite groups are strongly complete if and only if for each prime $p$ and each positive integer $d$ there exists an integer $b$ such that for any finite solvable group $G$ with a generating set of $d$ elements any element of the verbal subgroup $[G, G] G^{p}$ is of the form $\left[x_{1}, y_{1}\right] \cdots\left[x_{b}, y_{b}\right] z^{p}$ for some $x_{i}$, $y_{1}, z$ in $G$. This is an easy consequence of Proposition 4. Bounding the length of the derived sequence gives a similar result for solvable pro-finite groups.

We conclude with three examples.

(1) Let $\mathbf{Z}_{p}=\lim \mathbf{Z} /{ }_{p} n \mathbf{Z}$ be the additive group of $p$-adic integers and $\mathbf{F}_{l}\left[\left[\mathbf{Z}_{p}\right]\right]=\lim \mathbf{F}_{l}\left[\mathbf{Z} /_{p} n \mathbf{Z}\right]$ the continuous group ring of $\mathbf{Z}_{p}$ over the finite field $\mathbf{F}_{l}$ where $l$ is some rational prime. Then $Z_{p}$ acts continuously on $\mathbf{F}_{l}\left[\left[\mathbf{Z}_{p}\right]\right]$ with its profinite topology, and so we have a continuous semi-direct product

$$
\langle e\rangle \rightarrow \mathbf{F}_{l}\left[\left[\mathbf{Z}_{p}\right]\right] \rightarrow G \rightarrow \mathbf{Z}_{p} \rightarrow\langle e\rangle .
$$

$G$ is a two generator metabelian group and thus is strongly complete. $\quad \mathbf{F}_{l}\left[\left[\mathbf{Z}_{p}\right]\right]$ is an infinite $l$-torsion abelian group and thus is not strongly complete. Thus the profinite completion of the above exact sequence is not exact (compare with [1] Proposition 4).

(2) For $p$ a prime and $d$ a positive integer, let $G_{d, p}$ be the quotient of the free pro- $p$-group on $d$ generators by its second derived group. We have $\left[G_{d, p}, G_{d, p}\right] \cong \wedge^{1}\left(\mathbf{Z}_{p}\right)^{d}$. By dimension considerations we see that in order for the map

$$
\begin{gathered}
\left(G_{d, p} \times G_{d, p}\right)^{b} \rightarrow\left[G_{d, p}, G_{d, p}\right] \\
\left(x_{i}, y_{i}\right) \mapsto \Sigma\left[x_{i}, y_{i}\right]
\end{gathered}
$$

to be surjective we must have $2 d b \geqq d(d-1) / 2$ i.e. $b \geqq(d-1) / 4$. Now let $d_{n}$ be an unbounded sequence of positive integers and define $G=$ $\Pi_{n} G_{d_{n, p_{n}}}$ where $p_{n}$ denotes the $n$th prime. Then $G$ is a nilpotent group of type $(\mathrm{F})$ and thus is strongly complete. The commutator subgroup of $G$ is not closed. This may either be seen directly or by arguing as in the proof of Proposition 4. Note also that $\Pi_{n} G_{d_{n, p}}$ has a nonclosed commutator subgroup.

(3) Let $l$ and $p$ be primes and $d$ a positive integer. We define $G_{l, p, d}$ to be the semi-direct product of $\mathbf{F}_{p}^{d}$ with the augmentation ideal $I_{\mathbf{F}\left\{\mathbf{F}_{l}^{d}\right]}=\operatorname{Ker}\left(\mathbf{F}_{l}\left[\mathbf{F}_{p}^{d}\right] \rightarrow \mathbf{F}_{l}\right)$. Then the order of $G_{l, p, d}$ is $p^{d_{l} p^{d-1}}$ and any normal subgroup of $G_{l, p, d}$ has index divisible by $p$. One verifies by induction on 
$d$ that there exist elements of the group $\left[\mathbf{F}_{p}^{d}, \boldsymbol{I}_{\mathbf{F}\left[\mathbf{F}_{p}^{d}\right]}\right]$ which are products of no fewer than $d$ basic commutators.

Now fix $l$ and let $d_{n}$ be an unbounded sequence of positive integers. Let $G=\Pi_{n} G_{l, p_{n}, d_{n}}$ where $p_{n}$ is the $n$th prime different from l. Then $G$ is the semi-direct product of $A=\Pi_{n} \mathbf{F}_{p_{n}}^{d_{n}}$ with $B=\Pi_{n} I_{\mathbf{F}\left\{\mathbf{F}_{p n]}^{d n]} \text {. If }\right.}$ $G^{\prime}$ is a normal subgroup of $G$ of index $m$ such that $\left(p_{n}, m\right)=1$ for all $n \geqq N$, then $G^{\prime}$ contains $\Pi_{n \geqq N} G_{l, p_{n}, d_{n}}$. Thus $G$ is of type (F). One verifies as in example (2) that $[A, B]$ is not closed in $A$. Since $G /[A, B]=$ $B \times(A /[A, B])$, there exist discontinuous homomorphisms $G \rightarrow \mathbf{F}_{l}$. Thus $G$ is a metabelian profinite group of type (F) which is not strongly complete.

\section{REFERENCES}

1. M. P. Anderson, Exactness properties of profinite completion functors, Topology, 13 (1974), 229-239.

2. E. Hewitt and K. A. Ross, Abstract Harmonic Analysis I, Springer-Verlag, Heidelberg, 1963.

3. R. Kochendörffer, Group Theory, McGraw-Hill, London, 1970.

4. M. Lazard, Groups analytiques p-adiques, Publ. Math., I.H.E.S., no. 26 (1965).

5. W. Magnus, A. Karrass and D. Solitar, Combinatorial Group Theory, Interscience Publishers, New York, 1966.

6. H. L. Petersson, Discontinuous characters and subgroups of finite index, Pacific J. Math., 44 (1973), 683-691.

7. J. P. Serre, Cohomologie Galoisienne, Springer-Verlag, Heidelberg, 1965.

8. - Letter dated March 26, 1975.

Received February 25, 1975 and in revised form June 19, 1975. Partially supported by NSF grant GP-36418X1.

YALE UNIVERSITY 



\section{Pacific Journal of Mathematics}

\section{Vol. 62, No. $1 \quad$ January, 1976}

Mieczyslaw Altman, Contractor directions, directional contractors and

directional contractions for solving equations . .................. 1

Michael Peter Anderson, Subgroups of finite index in profinite groups .........

Zvi Arad, Abelian and nilpotent subgroups of maximal order of groups of odd order

John David Baildon and Ruth Silverman, On starshaped sets and Helly-type theorems ..........................................

John W. Baker and R. C. Lacher, Some mappings which do not admit an

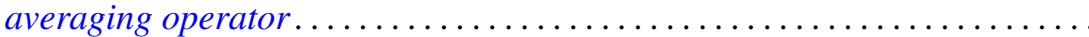

Joseph Barback, Composite numbers and prime regressive isols . . . . . . . . . .

David M. Boyd, Composition operators on $H^{p}(A) \ldots \ldots \ldots \ldots \ldots \ldots \ldots$

Maurice Chacron, Co-radical extension of PI rings . . . . . . . . . . . . .

Fred D. Crary, Some new engulfing theorems . . . . . . . . . . . . . . .

Victor Dannon and Dany Leviatan, A representation theorem for convolution transform with determining function in $L^{p} \ldots \ldots \ldots \ldots \ldots \ldots \ldots \ldots \ldots \ldots \ldots \ldots \ldots \ldots$

Mahlon M. Day, Lumpy subsets in left-amenable locally compact

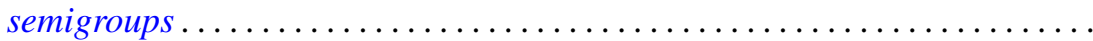

Michael A. Gauger, Some remarks on the center of the universal enveloping algebra of a classical simple Lie algebra . .

David K. Haley, Equational compactness and compact topologies in rings

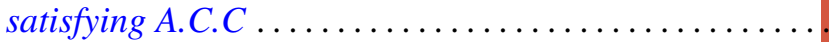

Raymond Heitmann, Generating ideals in Prüfer domains .

Gerald Norman Hile, Entire solutions of linear elliptic equations with

Laplacian principal part. .

Richard Oscar Hill, Moore-Postnikov towers for fibrations in which $\pi_{1}$ (fiber) is non-abelian

John Rast Hubbard, Approximation of compact homogeneous maps . .

Russell L. Merris, Relations among generalized matrix functions . .

V. S. Ramamurthi and Edgar Andrews Rutter, On cotorsion radicals ...

Ralph Tyrrell Rockafellar and Roger Jean-Baptiste Robert Wets, Stochastic

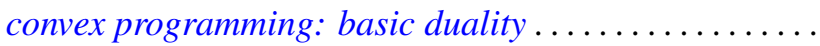

Alban J. Roques, Local evolution systems in general Banach spaces ..

I. Bert Russak, An indirect sufficiency proof for problems with bounded state variables.

Richard Alexander Sanerib, Jr., Ultrafilters and the basis property. .

H. A. Seid, The decomposition of multiplication operators on $L_{p}$-spaces . .

Franklin D. Tall, The density topology .................. 